Musées, Patrimoine et Culture scientifiques et techniques

$187 \mid 2020$

janvier-février 2020

\title{
Pôles, feu la glace. Exposer l'Anthropocène
}

\section{Ludovic Maggioni}

\section{OpenEdition \\ Journals}

Édition électronique

URL : http://journals.openedition.org/ocim/3536

DOI : 10.4000/ocim.3536

ISSN : 2108-646X

Éditeur

OCIM

Édition imprimée

Date de publication : 1 janvier 2020

Pagination : 28-35

ISSN : 0994-1908

Référence électronique

Ludovic Maggioni, « Pôles, feu la glace. Exposer l'Anthropocène », La Lettre de l'OCIM [En ligne], 187 |

2020, mis en ligne le 01 janvier 2021, consulté le 25 janvier 2021. URL : http://

journals.openedition.org/ocim/3536; DOI : https://doi.org/10.4000/ocim.3536

Ce document a été généré automatiquement le 25 janvier 2021.

Tous droits réservés 


\section{Pôles, feu la glace. Exposer l'Anthropocène}

\section{Ludovic Maggioni}

Visuel de l'exposition Pôles, feu la glace - création Anne Ramseyer.

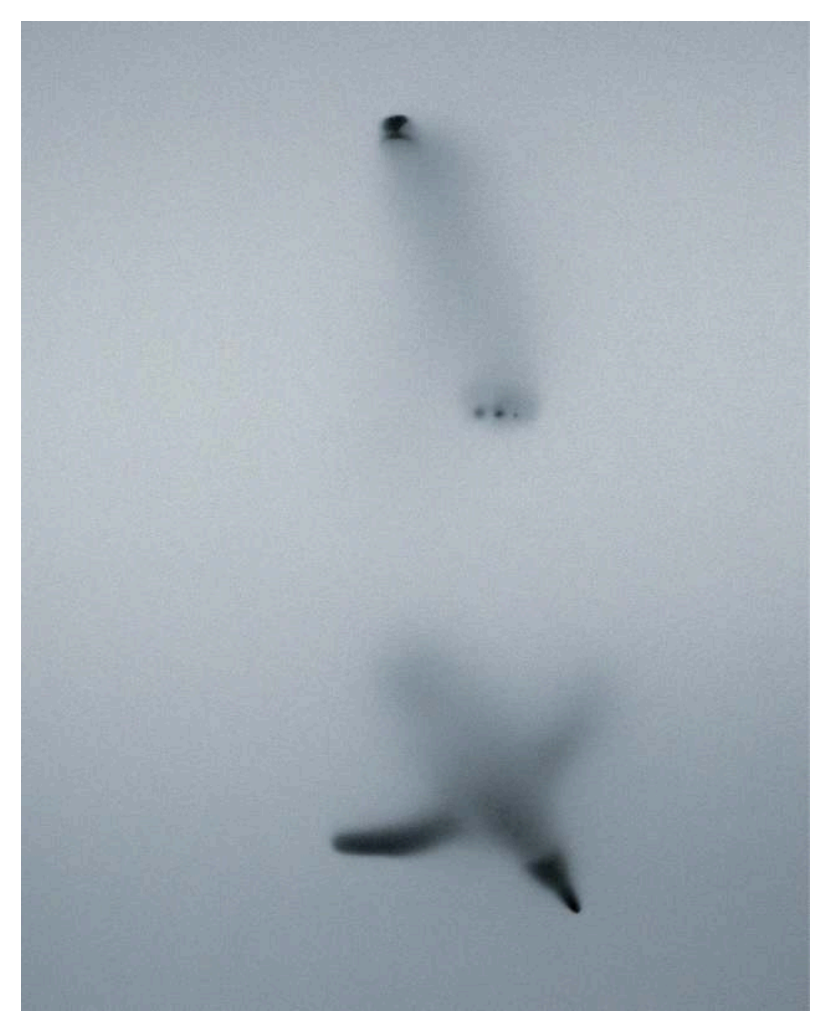

(c) MHNN

Situé dans une ville de 40000 habitants, le muséum d'Histoire naturelle de Neuchâtel dispose d'une riche collection réunie depuis le XVIII ${ }^{e}$ siècle. Au-delà du travail scientifique en lien avec la communauté internationale, il a pour ambition de créer des 
expositions temporaires sensibilisant le plus grand nombre aux relations que les humains entretiennent avec la nature. Pôles, feu la glace $e^{1}$, la dernière création, est un exemple de la manière dont un sujet de société peut être mis en scène par une équipe, en collaboration avec des scientifiques, des artistes et les publics.

\section{Un doux mensonge}

2 Très rapidement, il est apparu que le positionnement de l'exposition ne serait pas le climat et son changement. Partant du principe que les publics du muséum viennent pour partager un bon moment, compte tenu du battage médiatique sur les enjeux climatiques (moindre en 2016 au moment de la production, mais déjà latent), l'idée a été de mettre en place un projet autour de l'Arctique et de l'Antarctique. Ces territoires demeurent dans l'imaginaire collectif des lieux authentiques, où les règles de vie sont extrêmes, où tout est singulier. Ils ont un fort pouvoir d'attraction, mais sont également des terrains d'étude investis par les scientifiques du monde entier. De nombreuses recherches sont en cours, des études sur les climats du passé ont permis de mieux comprendre l'impact des humains sur la Terre. Le doux mensonge était créé, 50 $\%$ de l'exposition a été consacré au monde polaire: environnement, biologie, adaptations... et la moitié restante était dédiée au climat, à l'Anthropocène.

\section{Immersion polaire}

3 Sensibiliser, c'est, entre autres, faire vivre des expériences inoubliables. Pour ce faire, une invitation a été lancée au réalisateur de La marche de l'empereur : Luc Jacquet. L'idée que les visiteurs se trouvent au cœur d'une tempête dans une colonie de manchots empereurs a très vite émergé, la salle Ardemment glacial était née. Présenté dans un espace immersif, le film produit et réalisé par Luc Jacquet contenait des images montrant l'hyper-adaptation des manchots en mêlant vues de groupe, gros plans et images captées avec des caméras thermiques. La diffusion du son a également été investie par l'équipe technique pour renforcer le sentiment d'immersion. 
Ardemment Glacial, réalisé en collaboration avec Luc Jacquet. Cet espace permet une immersion dans une tempête au cœur d'une colonie de manchots empereurs.

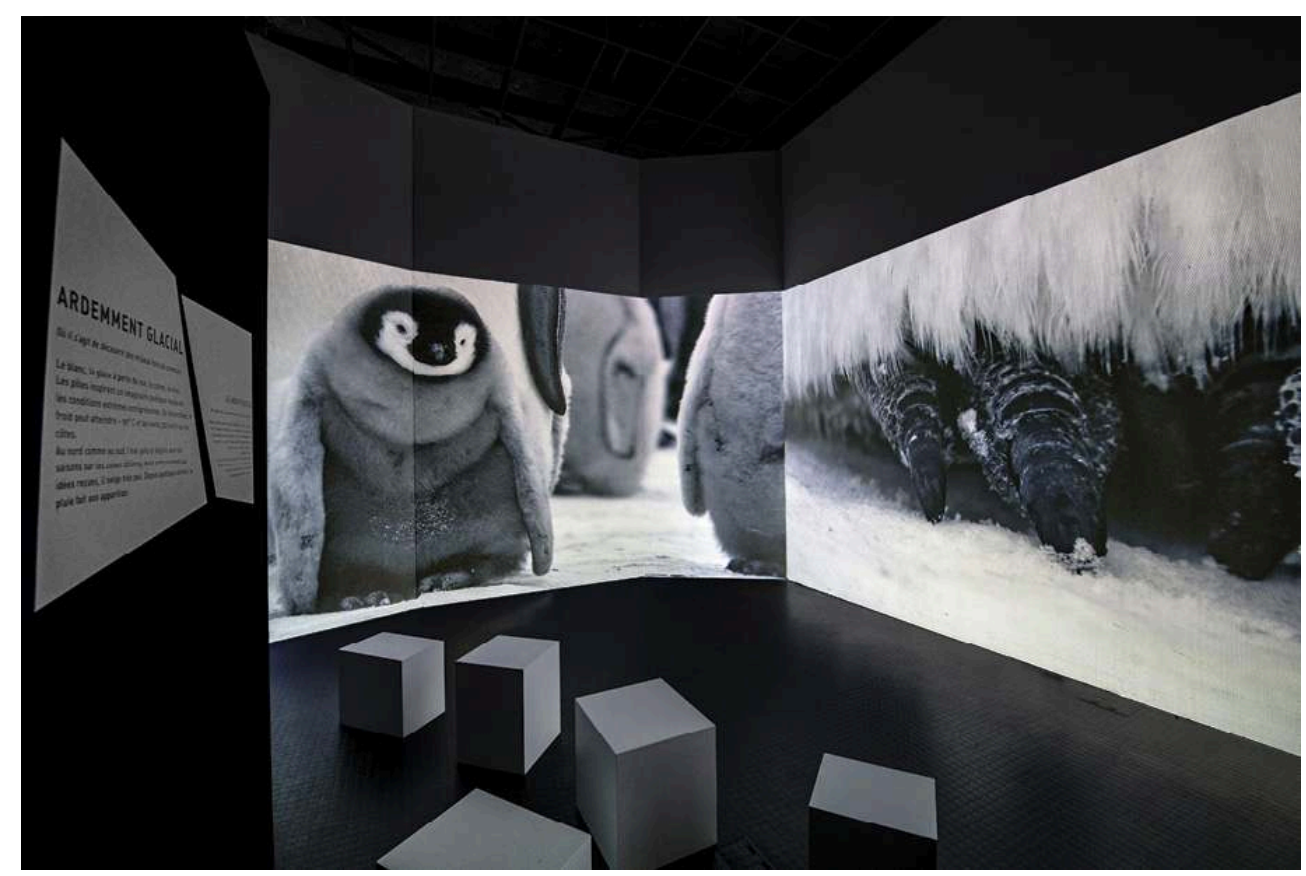

(c) Germond / MHNN

4 En parallèle à cet espace, une autre production du réalisateur a également été mise en scène. Dans la pièce sonore Effondrement, l'image s'efface au profit de l'ouïe. Le visiteur se retrouve dans une cabine noire de $10 \mathrm{~m}^{2}$ et entend les retentissements produits par l'effondrement d'un glacier en Antarctique durant un peu plus de 2 minutes. L'expérience sonore est troublante et joue sur l'imaginaire de la catastrophe chez les spectateurs.

\section{Parrainer l'Anthropocène}

$5 \quad$ La dimension scientifique présentant un focus sur le climat de la Terre est issue d'une rencontre avec Claude Lorius, glaciologue des pôles. La lecture de son ouvrage Voyage dans l'Anthropocène, cette nouvelle ère dont nous sommes les héros ${ }^{2}$, coécrit avec Laurent Carpentier, a été décisive dans la production. Relater l'importance des méthodes et des résultats des études menées par les scientifiques sur les glaces de l'Antarctique s'est alors révélé incontournable. Dans un souci de véracité, la salle Savoirs inconnus de l'exposition présentait une carotte de glace issue du pôle Sud - mise à disposition par l'Institut des géosciences de l'environnement de l'université Grenoble-Alpes-France mais aussi un Kraker, une machine employée par l'Institut de physique de Berne (Suisse) pour caractériser la composition gazeuse de la glace, ainsi qu'un graphique montrant les résultats de l'étude. La composition des bulles d'air contenues dans les glaces de l'Antarctique a permis de reconstituer les climats du passé de la Terre jusqu'à 800000 ans (forage Epica au Dôme en Antarctique) et de mettre en évidence l'impact des humains sur les climats de la Terre.

6 Malgré ces évidences, la notion d'Anthropocène fait toujours débat au sein de la Commission internationale de stratigraphie ${ }^{3}$. Pour les géologues, il demeure complexe 
de statuer sur une signature précise inscrite dans les couches de la roche marquant le début de cette nouvelle ère géologique. L'intention du muséum n'a alors pas été de traiter cette question de spécialiste, mais plutôt de se positionner sur le regard social porté sur l'Anthropocène : comment certains initiés et profanes perçoivent-ils ce sujet ?

7 Comme Claude Lorius avait accepté d'être le parrain de l'exposition, l'idée de créer une collection de témoignages sur la fonte des glaces a alors émergé. Un protocole de recueil a été mis en place et des témoins potentiels choisis en fonction des critères suivants : connaissance ou ignorance du sujet, internationalité, profession, âge... Au final ce sont plus d'une soixantaine de témoignages qui ont été recueillis. Ils ont été présentés dans la dernière salle de l'exposition Stars anonymes sans aucune intervention de la part de l'équipe de production et publiés dans un recueil: Votre intime conviction ${ }^{4}$ édité comme catalogue d'exposition.

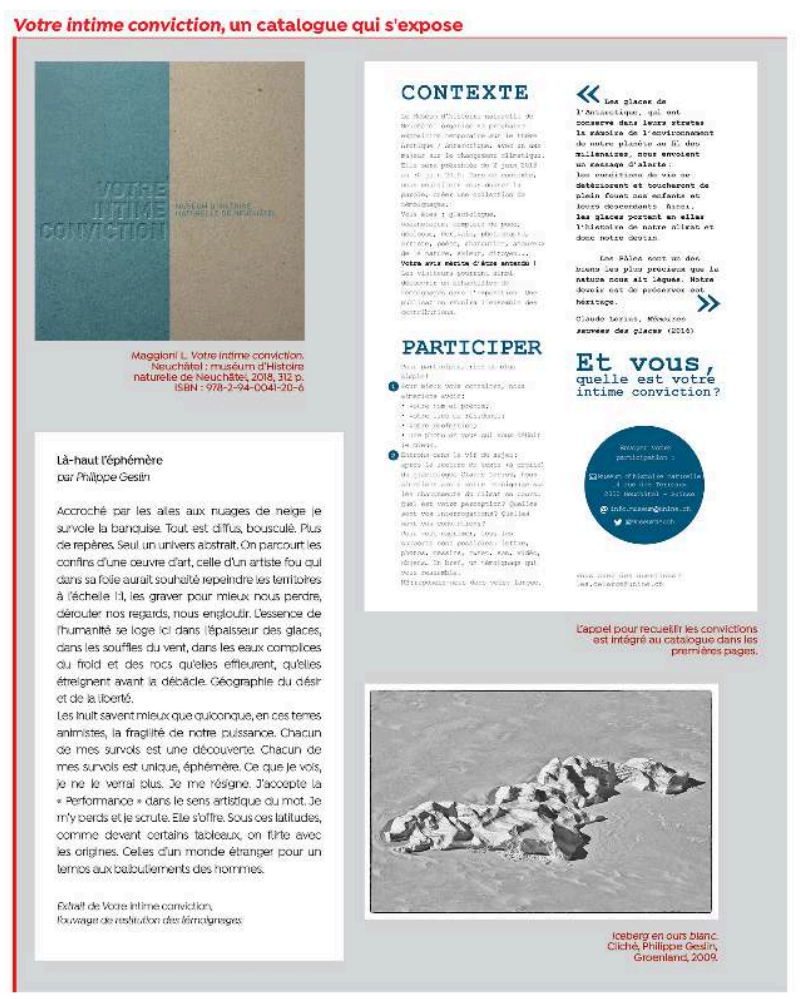


L'espace Savoirs inconnus présente une carotte deglace provenant d'Antarctique et un Kraker, machine utilisée par l'Institut de physique de Berne pour caractériser les gaz emprisonnés dans les glaces.

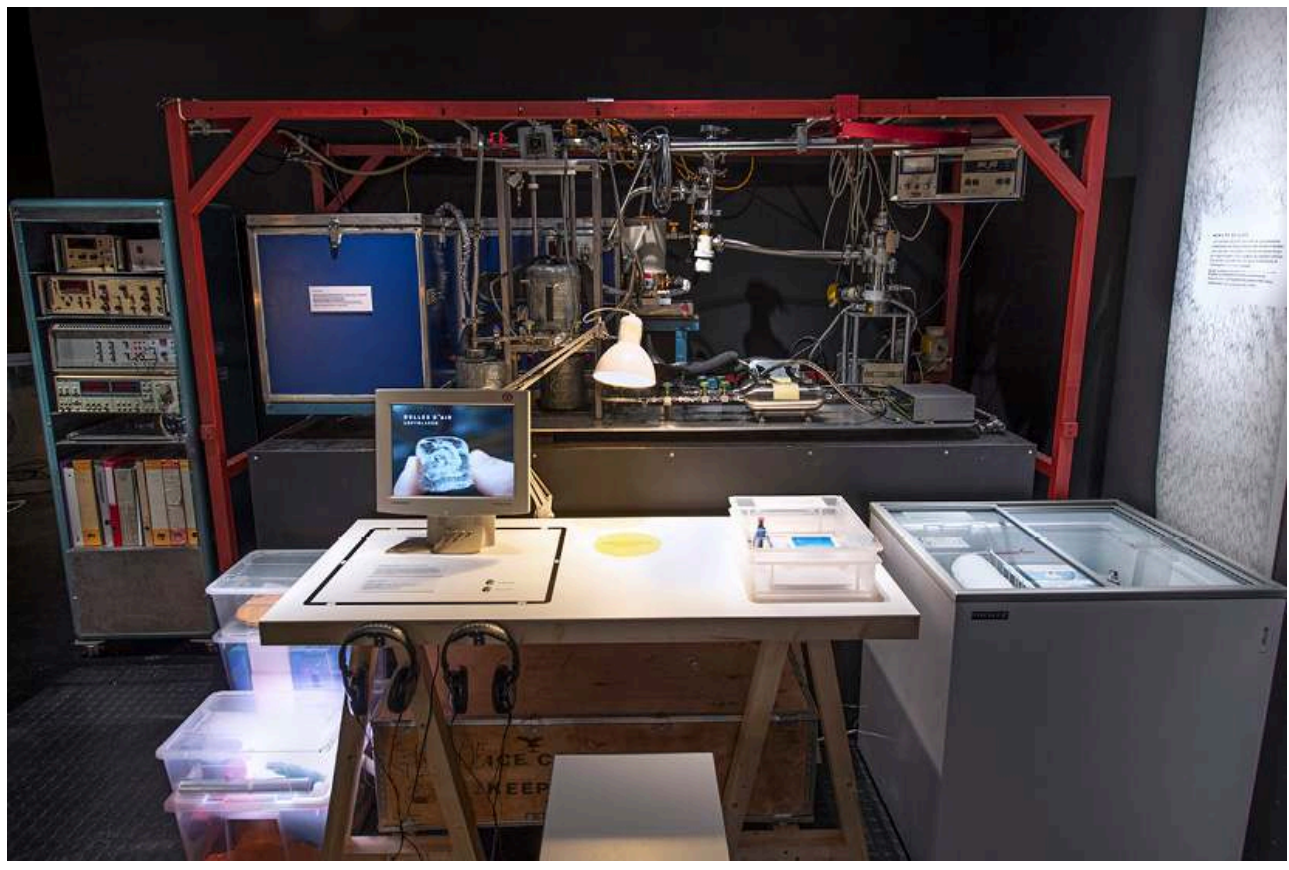

(c) Germond / MHNN

\section{Concilier délectation et alarmisme?}

La dramaturgie de l'exposition a été construite autour de la complexité de l'entendement, de la prise de conscience de l'Anthropocène par les sociétés humaines. Elle est écrite comme une sorte de voyage aux pôles, oscillant dans une tempête d'oppositions. Le parcours permet au visiteur des découvertes, des immersions, des surprises, bref des moments de vie variés et parfois forts en émotions. Le scénario oscille entre la fascination et des moments plus dramatiques, plus alarmistes. Il joue entre la grande Histoire et d'autres plus petites.

Par exemple, à mi-parcours, dans la salle Capharnaüm organisé, le visiteur peut découvrir les différents enjeux du changement climatique de manière chiffrée (les projections climatiques, le cycle du carbone, les accords de Paris, les $\mathrm{COP}^{5}$...) le tout environné d'horloges aux slogans plus ou moins alarmistes : "tempus fugit "; "courir après le temps »; « être dans l'air du temps »; " le temps c'est de l'argent »; " chercher midi à 14 heures »; "maintenant ou jamais». Soudainement le tic-tac des horloges s'emballe, la lumière s'éteint et une image de glacier s'embrase, le glas final retentit alors. Cet espace en changement d'état perpétuel propose une expérience plurisensorielle symbolique de l'urgence, de l'angoisse, de la difficulté à imaginer des futurs radieux. Pour dédramatiser et rendre accessible le contenu au plus grand nombre, des fictions convoquant des personnages célèbres ont été créées. Le Père Noël, la Reine des neiges et Jon Snow (célèbre personnage de la série Game of Thrones), livrent leurs points de vue sur le changement climatique. Ainsi, le Père Noël est redevenu vert et a dû déménager en Antarctique pour cause de disparition de l'Arctique, la Reine des neiges est une fan inconditionnelle des accords sur le climat mais doit préserver son palais de 
glace et Jon Snow doit gérer des réfugiés climatiques. Ces courtes fictions tournées avec un unique comédien en ont fait sourire plus d'un.

Par la suite, dans un interlude nommé Feu la glace, le visiteur se trouve face à un immense iceberg, éclairé d'une lumière divine. Il découvre les courbes de projection de fonte des calottes arctiques et antarctiques. À droite trône un reliquaire givré dans lequel sont conservés les derniers morceaux de glace terrestre. Au-dessus, un faire-part de décès annonce sa disparition. En face des glaces (esquimaux, cornets...) fondent sur un grand écran en accéléré. À ce jour, cette fiction de sépulture devient réalité. Des glaciers disparaissent et font l'objet de funérailles symboliques comme dernièrement celui du Pizol (Alpes suisses) le 22 septembre $2019^{6}$.

\section{Une utopie scénographique}

Aux confins du monde, les pôles. Au bout de nos rêves, l'ours polaire et le manchot empereur.

Le changement climatique, le réchauffement de la planète, l'impact humain sur l'évolution de la Terre...

Feu la glace.

Et si la scénographie collait littéralement à son sujet?

Les glaces se sont mises à fondre dramatiquement, l'exposition pourrait fondre elle aussi, se résorber, disparaître, se dissoudre.

"Au commencement serait le blanc, la glace, le froid, les animaux, les gens, les objets, les textes, les explications, les commentaires, les points de vue.

Au fur et à mesure du parcours, un peu moins de tout. Imperceptiblement les objets se feraient plus rares, les textes plus courts et un peu moins lisibles, les animaux moins présents. Le ruissellement de l'eau couvrirait les craquements de la glace. En chemin, les architectures s'amenuiseraient, les espaces disparaitraient pour se confondre.

Le public, dans un premier temps, ne comprendrait peut-être pas ce léger dérangement, ce glissement vers le moins. Un malaise qui s'installerait, et des questions qui surgiraient : estce que c'est voulu? Est-on dans un chantier? L'exposition n'est pas terminée ? Pourquoi si peu de lumière? Et tout ce vide? Il n'y a plus qu'un mot sur deux? On nous maltraite! Où est la sortie?

On poursuivrait sur le mode de la privation. De blanche, l'exposition deviendrait noire.

Vider graduellement une exposition de ce qui la constitue? Un pari trop culotté?

Et à la fin? Une touche d'espoir. Oui ? Ou non?»

De ce rêve scénographique, excessif peut-être, et bien plus difficile à réaliser qu'il n'y paraît, de ce désir de cohérence extrême, ont découlé de belles idées qui se sont glissées dans la réalité. Une utopie inspirante, à la source notamment de la salle du Capharnaüm organisé dans laquelle la scénographie ne s'est pas édifiée, mais bien dé-construite dans un espace habité, sur les vestiges de l'exposition 
précédente. Dans ce décor de ruines à caractère dramatique, le public est saisi dans un cycle dynamique de sons et de lumières qui se veut déroutant, voire oppressant, et qui entrave délibérément le cours de sa visite.

Une goutte d'eau tombe du plafond, persistante. Ailleurs un sol de glace se dilue peu à peu dans la mer, ou ailleurs encore, les images chocs d'une station baleinière abandonnée ${ }^{7}$ marquent un point de bascule à partir duquel le ton donné à

l'exposition se modifie. L'état d'urgence est déclaré. Alerté jusqu'à l'effondrement final du glacier, le public quittera l'exposition sous le regard indolent de l'ours polaire en balade sur sa banquise...

Anne Ramseyer

Scénographe au muséum d'Histoire naturelle de Neuchâtel - 2019

Capharnaüm organisé, un espace déconstruit de l'exposition précédente.
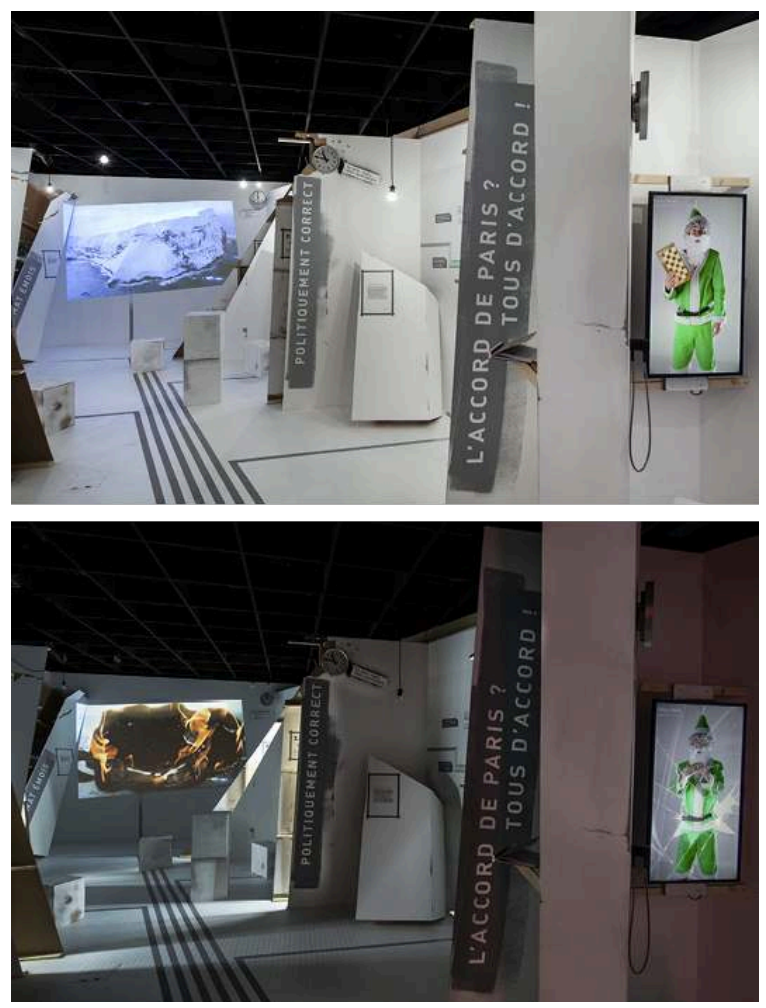

(c) Germond / MHNN

\section{Le jour d'après}

11 La capacité de nos sociétés à se projeter dans un futur réjouissant est aujourd'hui nébuleuse. Afin de prolonger l'exposition, la programmation culturelle a exploré ce sujet. Pour ce faire, une Nuit de la débâcle a été mise en place le 18 mai 2019 en partenariat avec le NIFFF (Neuchâtel international fantastic film Festival). Dans des tentes, dans une ambiance post-apocalyptique, des films et courts métrages de sciencefiction ont été projetés dans le cadre de la Nuit des musées. Pour ne pas rester sur des 
idées sombres le lendemain, le public était convié à vivre «le jour d'après ». Cette journée était axée sur les organisations alternatives en offrant aux collectifs, associations et start-up locales des espaces au cœur du muséum. Un vide-dressing a ainsi côtoyé une gratiferia ${ }^{8}$, un repair cafég ${ }^{9}$, un marché bio, une société commercialisant des pailles comestibles... Autant d'acteurs et de publics pour vivre un lendemain radieux et souligner les potentialités offertes par le changement.

Dans l'interlude Feu la glace, on peut observer le reliquaire de glace terrestre à droite, et au-dessus le faire-part de décès. À gauche, le film d'une glace esquimau qui fond au ralenti.
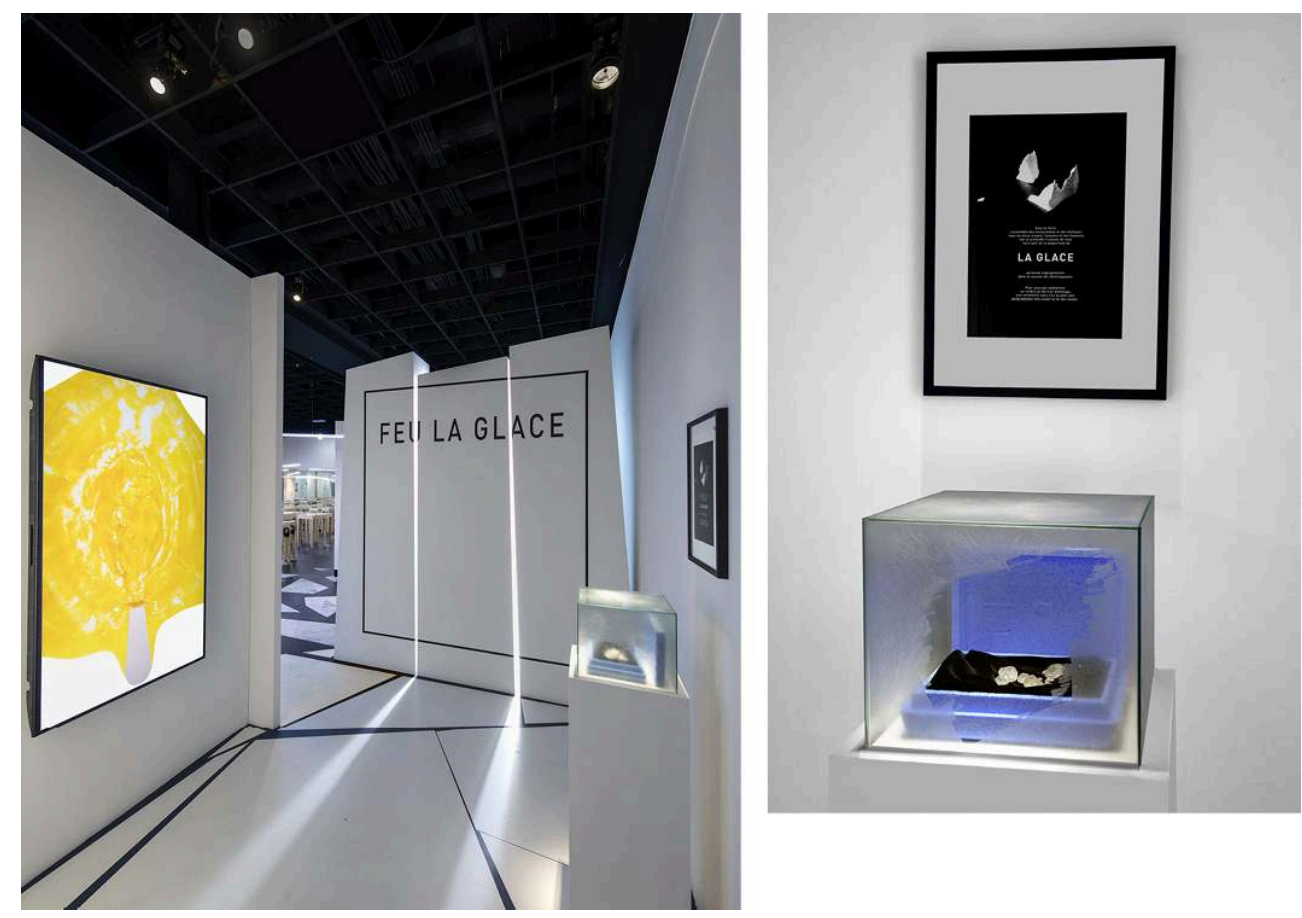

(C) Germond / MHNN 
Stars anonymesest l'espace de restitution des témoignages recueillis sur la disparition des glaces en hommageà Claude Lorius.

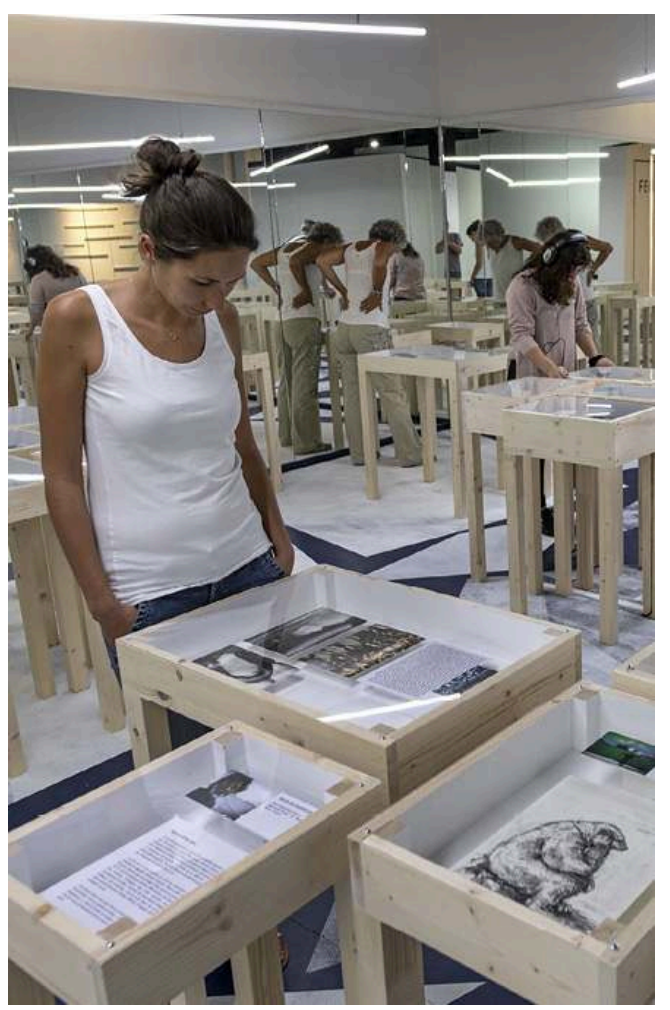

(C) Germond / MHNN 
Dans la salle Capharnaüm organisé, les prises de parole en vidéo de personnages iconiques (comme ici, Jon Snow) permet d'ajouter un peu de légèreté à l'exposition et de donner le sentiment que le sujet est accessible à tous les publics.

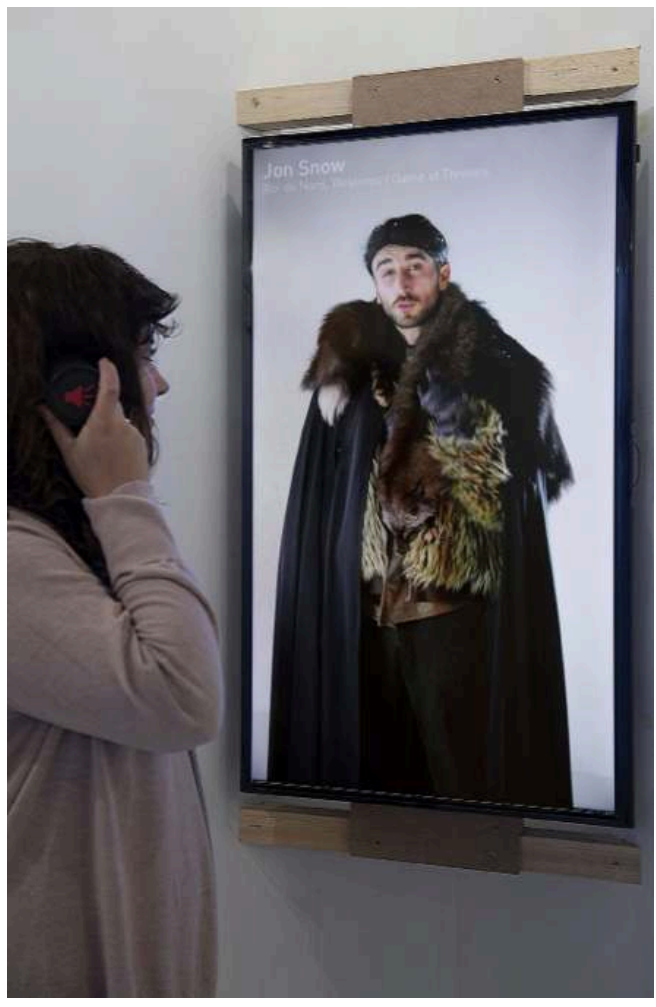

(c) DR-MHNN

\section{Muséologie sensible}

Les dispositifs développés dans l'exposition l'ont été avec le désir de stimuler les émotions, de la joie à la tristesse. Une muséologie qualifiée de sensible est un outil qui renforce le sentiment d'appartenance et d'engagement des publics comme le montrent très certainement ces messages recueillis dans le livre d'or : "Cette expo m'a fait ouvrir les yeux, c'est comme un coup de gueule qui fait du bien!» Malou ; "Merci pour cette belle expo qui nous parle, qui parle à nos yeux, oreilles, cœur. Toujours aussi agréable et créatif: Bravo.» Séverine.

13 Cette expérience souligne l'importance du rôle que les muséums peuvent et doivent jouer en tant que point de jonction entre sciences et société. La mise en culture de l'Anthropocène n'est pas uniquement un enjeu scientifique mais il est aussi social. Au cœur de la controverse, le musée devient un lieu refuge où les rencontres, les informations sont accessibles, où les modèles sociaux émergents peuvent être mis en lumière. 


\section{NOTES}

1. Pôles, feu la glace est une exposition du muséum d'Histoire naturelle de Neuchâtel présentée du 9 septembre 2018 au 18 septembre 2019.

2. Carpentier L. Voyage dans l'Anthropocène, cette nouvelle ère dont nous sommes les héros. Arles: éditions Actes Sud, 2011, 168 p.

3. Site Internet de la Commission internationale de stratigraphie : www.stratigraphy.org

4. Maggioni L. et al. Votre intime conviction. Neuchâtel : Muséum d'Histoire naturelle de Neuchâtel, 2018, 312 p.

5. La Conférence des parties (ou Conférence des États signataires) est l'organe qui vérifie la bonne application des objectifs des conventions internationales adoptées en matière environnementale. Elle est convoquée au moins une fois tous les deux ans par le ou la directrice de l'Unesco.

6. Pedrero A., Rouyer E. En Suisse, des funérailles en montagne pour un glacier disparu, AFP, 22 septembre 2019.

7. Vidéo de Simon Faithfull. Titre: We climbed round a final ridge and saw a whaling-boat enetring the bay 2500ft, below. Durée : 00:11:54 ; 2005. Prêt : Frac Franche-Comté.

8. Imaginé en 2010 par l'Argentin Ariel Rodríguez Bosio pour répondre à une situation d'encombrement d'objets devenus inutiles, il s'agit d'une zone de gratuité éphémère sur laquelle les participants peuvent déposer leurs dons ou se servir librement sans contrepartie.

9. Atelier consacré à la réparation d'objets et organisé à un niveau local, souvent sous forme de tiers-lieu.

\section{RÉSUMÉS}

L'objectif de l'exposition Pôles, feu la glace était d'expérimenter des moyens de mise en culture de la notion d'Anthropocène. Comment créer une exposition originale; concilier culpabilité, alarmisme et plaisir; et aborder les débats de société que suscite le sujet sans faire fuir les publics? Retour sur la philosophie générale du projet et les dispositifs utilisés pour mettre en lumière l'action de l'humain sur la planète.

\section{INDEX}

Mots-clés : développement durable, exposition temporaire, grande institution muséale nationale

\section{AUTEUR}

\section{LUDOVIC MAGGIONI}

Directeur du muséum d'Histoire naturelle de Neuchâtel

ludovic.maggioni@unine.ch 